\title{
Méthode de calcul de l'évolution de l'interaction d'un tourbillon et d'une houle
}

\author{
S. Mordane*, G. Mangoub**, M. Chagdali*** \\ * Maitre assistante, Faculté des Sciences Ben M'Sik, L.C. S. M Casablanca \\ **Maitre assistante, Faculté des Sciences et Techniques Settat, Maroc \\ *** Professeur, Faculté des Sciences Ben M'Sik, L.C. S. M Casablanca
}

\section{Résumé:}

On présente une étude numérique sur le comportement d'une concentration tourbillonnaire dans la houle. Le tourbillon concentré peut être dû aussi bien à la présence d'obstacles naturels qu'artificiels, comme par exemple les socles d'ouvrages. Nous traitons séparément le calcul de la houle et de la surface libre d'une part et celui de l'évolution du tourbillon d'autre part. Nous ne considérons pas la génération du tourbillon: le fond est choisi plat. Le but est de développé un code de calcul numérique qui permet de suivre l'évolution d'une concentration tourbillonnaire dans le champs de la houle non linéaire.

\begin{abstract}
:
We present a numerical study about the comportment of concentrated vortical in Waves. The concentrated vortical can be had to the natural obstacle presence as well as artificial, as for example pedestals of works. We process separately the calculation of the waves and the free surface on the one hand and the evolution of the vortex on the other hand. We don't consider the generation of the vortex: the bottom is chosen flat. The purpose is to develop a numerical code that allows to follow the evolution of concentrated vortical in the fields of the nonlinear waves.
\end{abstract}

\section{Introduction:}

On se propose donc d'étudier numériquement l'évolution du tourbillon dans le champs de houle. La présence d'obstacle dans la houle génère des lâchages tourbillonnaires qui peuvent avoir des impacts sur le fond. Ces impacts se manifestent par des phénomènes d'érosion, d'envasement et de transport des sédiments. Il est donc important de connaître et d'étudier ces zones d'impacts qui sont lié à l'évolution tourbillónnaire. Lors de la résolution numérique d'un tel type de problème par des méthodes classiques (différences finies, élément finies), on se confronte à un certain nombre de difficulté [1], [2]. Il est possible d'utiliser le couplage de différents méthodes numériques. Dans ce travail, on a couplé la méthode des différences finies, pour le calcul de la houle et la méthode particulaire pour le calcul du tourbillon. Nous ne considérons pas la génération du tourbillon et la houle utilisée est obtenue numériquement. Dans le but de clarifier la démarche utilisée, on va exposer respectivement le calcul de la houle numérique, le calcul de l'évolution du tourbillon et enfin le problème du couplage houle-tourbillon. 


\section{Calcul de la houle numérique:}

Nous nous intéressons à la simulation de la propagation de la houle dans un canal numérique, dans l'approximation fluide parfait. L'approximation de fluide parfait, incompressible et irrotationnel permet de définir le potentiel des vitesses $\varphi(x, y, t)$. Les difficultés de ce problème proviennent essentiellement du calcul de la déformée de la surface libre non-linéaire et de l'écriture des conditions d'absorptions en aval du domaine de calcul. Dans ce travail on utilise un système de coordonnées curvilignes qui permet d'épouser la forme de la surface libre à chaque instant et de tenir compte de l'irrégularité du fond. Cette transformation facilite l'écriture des conditions aux limites sur les frontières irrégulières et évolutives du domaine d'étude. Ainsi le problème physique sera transformé dans un domaine rectangulaire, figé dans le temps et dans l'espace.

\subsection{Formulation du problème de la houle dans le domaine physique:}

Nous considérons l'écoulement plan et irrotationnel d'un fluide parfait et incompressibie dans un canal à houle de longueur $\mathrm{L}$ et de hauteur moyenne $\mathrm{H}$. Le fluide est mis en mouvement sous l'action d'un batteur en amont. En aval, on suppose que la houle est absorbée (figurel).

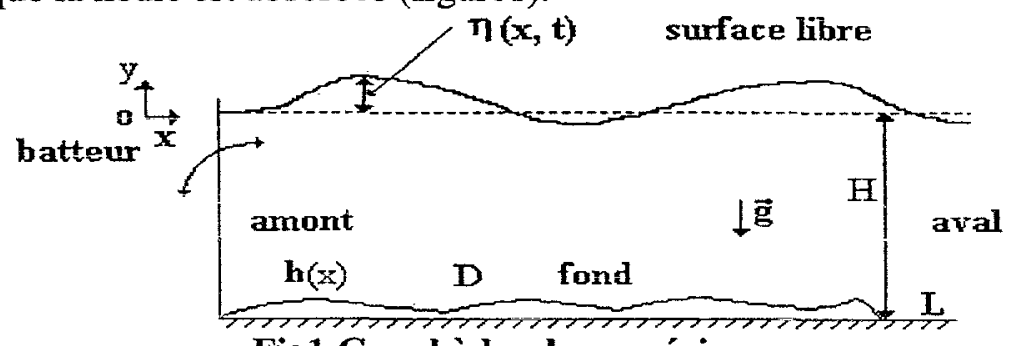

Figl Canal à houle numérique

Les équations non linéaires de la houle s'écrivent en terme du potentiel des vitesses $\varphi(\mathrm{x}, \mathrm{y}, \mathrm{t})$ et d'élévation de la surface libre $\eta(\mathrm{x}, \mathrm{t})$ en variables cartésiennes sous la forme:

$$
\begin{array}{cr}
\frac{\partial^{2} \varphi}{\partial x^{2}}+\frac{\partial^{2} \varphi}{\partial{ }^{2}}=0 & \text { dans D domaine d'écoulement } \\
\frac{\partial \varphi}{\partial \mathrm{y}}+\frac{\partial \varphi}{\partial \mathrm{x}} \frac{\partial \mathrm{h}}{\partial \mathrm{x}}=0 & \text { sur le fond } ; \mathrm{y}=\mathrm{h}(\mathrm{x}) ; 0 \leq \mathrm{x} \leq \mathrm{L} \\
\frac{\partial \varphi}{\partial t}+0.5\left(\left(\frac{\partial \varphi}{\partial x}\right)^{2}+\left(\frac{\partial \varphi}{\partial y}\right)^{2}\right)+g \eta=0 & \text { sur la surface libre } \mathrm{y}=\eta(\mathrm{x}, \mathrm{t}) ; 0 \leq \mathrm{x} \leq \mathrm{L} \\
\frac{\partial \eta}{\partial t}+\frac{\partial \varphi}{\partial x} \frac{\partial \eta}{\partial x}-\frac{\partial \varphi}{\partial y}=0 & \text { sur la surface libre } \mathrm{y}=\eta(\mathrm{x}, \mathrm{t}) ; 0 \leq \mathrm{x} \leq \mathrm{L}
\end{array}
$$

L'existence d'un générateur de houle correspond à des conditions aux limites sur le potentiel des vitesses de la forme:

$$
\varphi=\varphi_{\text {amont }}=a f_{b}(y, t) \quad x=0 ; h(x) \leq y \leq \eta(0, t)
$$

La fonction $f_{b}(y, t)$, connue à priori, contient toutes les informations sur les caractéristiques de la houle (sa période et son vecteur d'onde).

Ce problème tel qu'il est proposé, présente essentiellement les difficultés suivantes: problème non linéaire, instationnaire, écrit sur un domaine à frontières courbes et évolutif et à frontières ouvertes en aval. 


\subsection{Formulation du problème dans un système de coordonnées curvilignes:}

L'utilisation du système de coordonnées curvilignes suivant:

$$
X=\mathrm{x}, Y=\frac{\mathrm{y}-\mathrm{h}(\mathrm{x})}{\eta(\mathrm{x}, \mathrm{t})-\mathrm{h}(\mathrm{x})}, \mathrm{t}=\mathrm{t}
$$

permet de résoudre une partie des difficultés cités précédemment, à savoir l'écriture des conditions aux limites sur la frontière courbe et évolutif de la surface libre. Ainsi on ramène le problème précèdent écrit dans le domaine physique (D) en un problème transformé écrit dans un domaine rectangulaire figé dans le temps et dans l'espace (D') (voir figure 2).

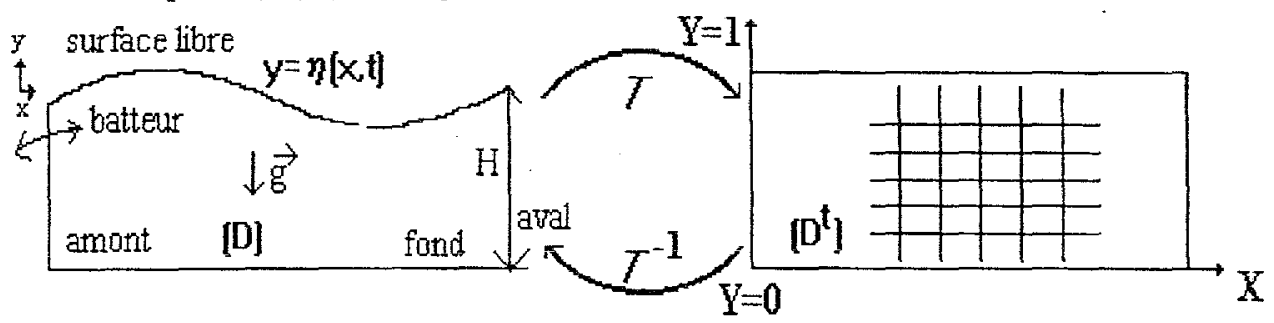

Figure (2)

Les équations du mouvement dans le plan transformé $(X, Y)$ s'écrivent sous la forme:

$$
\begin{aligned}
& \frac{\partial^{2} \varphi}{\partial X^{2}}+(f(X))^{2} \frac{\partial^{2} \varphi}{\partial Y^{2}}+(g(X))^{2} \frac{\partial^{2} \varphi}{\partial Y^{2}}+2 g(X) \frac{\partial^{2} \varphi}{\partial X \partial Y}+\frac{\partial g(x)}{\partial X} \frac{\partial \varphi}{\partial Y}=0 \text { dans } D^{t} \\
& g(X) \frac{\partial \varphi}{\partial \mathrm{Y}}+\frac{\partial h}{\partial \mathrm{X}}\left(\frac{\partial \varphi}{\partial \mathrm{X}}+\mathrm{f}(\mathrm{X}) \frac{\partial \varphi}{\partial \mathrm{Y}}\right)=0 \quad \text { sur le fond } ; \mathrm{Y}=0,0 \leq \mathrm{X} \leq \mathrm{L} \\
& \frac{\partial \varphi}{\partial \mathrm{t}}-\frac{1}{\mathrm{P}(\mathrm{x})} \frac{\partial \varphi}{\partial \mathrm{Y}} \frac{\partial \eta}{\partial \mathrm{t}}+0.5\left(\left(\frac{\partial \varphi}{\partial \mathrm{x}}+\mathrm{g}(\mathrm{x}) \frac{\partial \varphi}{\partial \mathrm{Y}}\right)^{2}+\left(\mathrm{f}(\mathrm{x}) \frac{\partial \varphi}{\partial \mathrm{Y}}\right)^{2}\right)+g \eta=0 \quad \text { sur } \mathrm{Y}=1 ; 0 \leq \mathrm{X} \leq \mathrm{L} \\
& \frac{\partial \eta}{\partial \mathrm{t}}+\left(\frac{\partial \varphi}{\partial \mathrm{x}}+\mathrm{g}(\mathrm{x}) \frac{\partial \varphi}{\partial \mathrm{Y}}\right) \frac{\partial \eta}{\partial \mathrm{x}}=\mathrm{f}(\mathrm{x}) \frac{\partial \varphi}{\partial \mathrm{Y}} \\
& \varphi=\varphi_{\text {amont }}=a f_{b t}(y, t) \\
& \text { sur } \mathrm{Y}=1 ; 0 \leq \mathrm{X} \leq \mathrm{L}(4) \\
& \mathrm{X}=0 ; 0 \leq \mathrm{Y} \leq 1
\end{aligned}
$$

Avec:

$$
\mathrm{g}(\mathrm{x})=\frac{\mathrm{Y}-1}{\mathrm{P}(\mathrm{x})} \frac{\partial \mathrm{h}}{\partial \mathrm{x}}-\frac{\mathrm{Y}}{\mathrm{P}(\mathrm{x})} \frac{\partial \eta}{\partial \mathrm{x}}, \mathrm{f}(\mathrm{x})=\frac{1}{\mathrm{P}(\mathrm{x})}, \mathrm{P}(\mathrm{x})=\eta(\mathrm{x}, \mathrm{t})-\mathrm{h}
$$

C'est ce problème qui fera l'objet de notre étude numérique, où on s'attachera de résoudre les deux difficultés suivantes: l'écriture des conditions aux limites en aval et le traitement numérique des termes non linéaire.

1.3 Résolution numérique: Pour la résolution numérique, on a choisi la méthode des différence finies. On expose par la suite le calcul du champs potentiel, des conditions limites à la surface libre et le traitement des conditions en aval.

1.3.1 Calcul du potentiel à l'intérieur: La résolution de l'équation elliptique (1) permet d'accéder au potentiel des vitesses à l'intérieur du domaine de calcul. La méthode choisie est basée sur le schéma des directions alternées implicite proposée par Douglas [4]. Pour son application aux équations elliptiques, on confère d'abord à l'équation considérée un caractère parabolique, par l'adjonction d'un terme d'évolution fictif. Ensuite, on adopte le schéma de Douglas tel qu'il est proposée dans la référence [4]. Les itérations internes sont réalisées en utilisant les coefficient optimum de convergence défini dans [5]. 
1.3.2 Calcul des conditions aux limites sur la surface libre: Ces conditions aux limites sont écrites sur la ligne $Y=1$. Elles sont discrétisée par un schéma de Cranck-Nicholson à l'ordre deux. Les termes non linéaires sont linéarisés dans le temps. Les termes où apparaissent les dérivées par rapport à $Y$ sont couplés fortement avec la solution intérieur déduit du champs elliptique, moyennant la procédure des itérations internes.

1.3.3 Traitement des conditions en aval: La difficulté d'écrire les conditions aux limites à l'aval vient essentiellement du manque d'information sur l'extérieur du domaine de calcul. Pour surmonter cette difficulté on écrit les conditions de radiation de type Sommerfield:

$$
\frac{\partial \varphi}{\partial t}+\mathrm{F} \frac{\partial \varphi}{\partial \mathrm{x}}=0 \quad \text { en aval } ; \mathrm{x}=\mathrm{L} ; \mathrm{h}(\mathrm{x}) \leq \mathrm{y} \leq \eta(\mathrm{L}, \mathrm{t})
$$

où $\mathrm{F}$ est la vitesse de phase de propagation de l'onde qui est déterminée à partir de trois niveaux de temps et des points au voisinage de la frontière [6].

\section{4_Résultats:}

On suppose que la houle incidente, générée par le batteur est de la forme:

$$
\mathrm{a} \hat{\mathrm{f}}_{\mathrm{b}}(\mathrm{y}, \mathrm{t})=\mathrm{a} \frac{\mathrm{g}}{\mathrm{w}} \frac{\mathrm{ch}(\mathrm{K} \cdot \mathrm{y})}{\operatorname{ch}(\mathrm{K} \cdot \mathrm{h})} \sin (-\mathrm{wt}) ; \mathrm{t}>0
$$

On choisit la pulsation $\mathrm{w}=2 \Pi / \mathrm{T}=3.18 \mathrm{~s}^{-1}$. On prendra la pesanteur $\mathrm{g}=9.81 \mathrm{~ms}^{-1}$. Le vecteur d'onde $\mathrm{K}$ correspondant est calculé à partir de la relation de dispersion

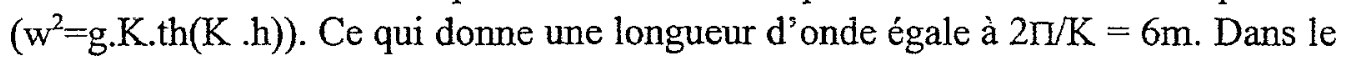
but de valider le code de calcul, on se place dans un canal à houle de profondeur constante $\mathrm{h}=2.5 \mathrm{~m}$ et de longueur $\mathrm{L}=21 \mathrm{~m}$. On présente respectivement, la propagation de la houle, la validation des conditions aux limites et la confrontation avec la théorie classique de stokes aux différents ordres.

Sur la figure 3-a, on présente la propagation de l'onde de gravité à la surface libre pour une amplitude du batteur en amont égale à $0.01 \mathrm{~m}$ au bout d'un quart de période. Dans tous ce paragraphe on notera $\mathbf{X}$ la longueur du canal $(\mathrm{m}), \eta$ la dénivellation de la surface libre $(m)$ et iso $\varphi$ les isovaleurs du potentiel.

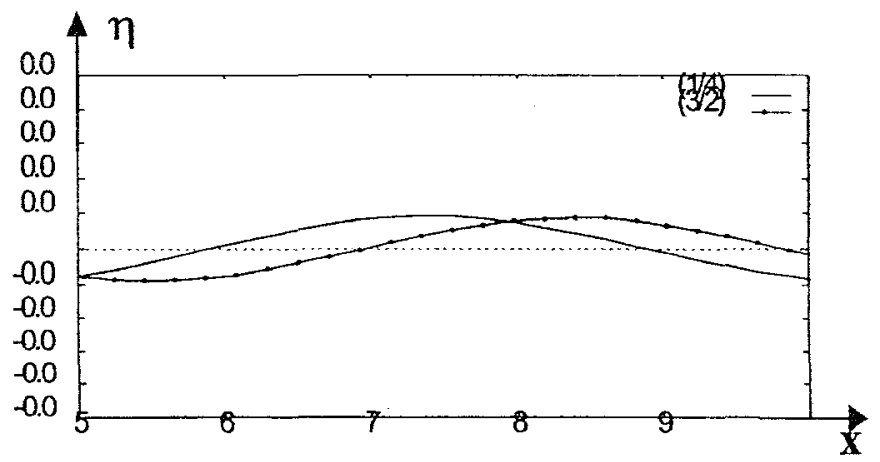

Figure 3-a Propagation de la houle le long du canal

Sur la figure 3-b, on donne une présentation tridimensionnel des iso-valeurs du potentiel des vitesses dans tout le champs physique. 


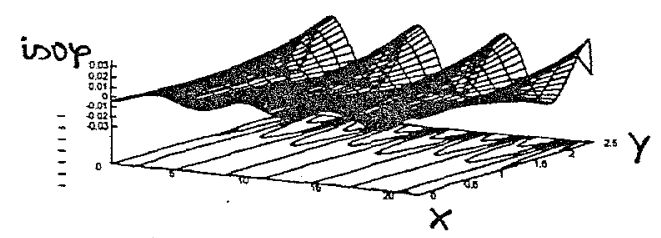

Figure 3-b Présentation tridimensionnelle du potentiel

Vue ces résultats, on constate une bonne progression de l'élévation de la surface libre avec une conservation de la masse d'eau le long de la ligne moyenne de la surface au repos. Sur les figures 4-a et 4-b, on présente une validation des conditions aux limites en aval. Pour cela, on a choisit deux domaines de tailles différentes et on a comparé, respectivement l'élévation de la surface libre et les iso-valeurs du potentiel des vitesses à l'intérieure du domaine du calcul. La figure 4-a présente la propagation de la houle pour un domaine de longueur $21 \mathrm{~m}$ et pour un domaine de longueur $12 \mathrm{~m}$.

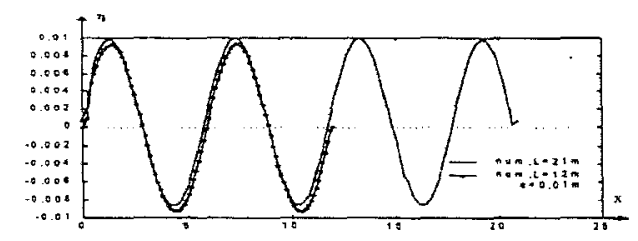

Figure 4-a Validation des conditions en aval

On peut constater, sur la figure 4-a, qu'il y a une légère différence (de l'ordre de 7/1000) qui vient certainement des conditions aux limites en aval. Une présentation par les iso-valeurs du potentiel des vitesses (figure 4-b) confirme cette constatation.

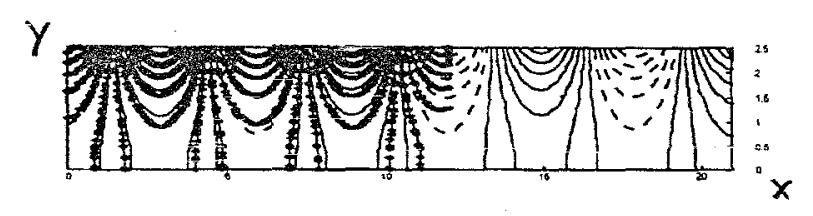

Figure 4-b Validation des conditions en aval

On présente par la suite, une confrontation de nos résultats numériques avec ceux de la théorie de Stokes à l'ordre deux. La comparaison avec Stokes à l'ordre deux est faite pour une amplitude $a=0.1 \mathrm{~m}$ (figure $5-\mathrm{a}$ ) et avec Stokes linéaire (ordre un) pour $\mathrm{a}=0.15$ (figure 5-b). Cela correspond respectivement à des cambrures de l'ordre de $0.016(\mathrm{a}=0.1 \mathrm{~m})$ et $0.025(\mathrm{a}=0.15 \mathrm{~m})$.
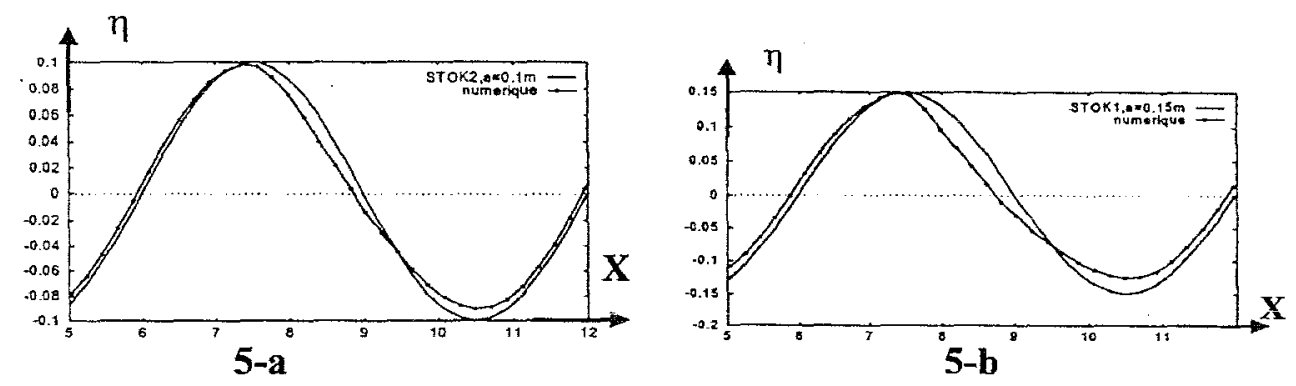

Figure 5 Confrontation numérique - théorie de Stokes. 
On constate sur la figure 5-b (comparaison numèrique-thèorie linéaire de Stokes) qu'il y a une dissymétrie amont aval au niveau de la forme de chaque crête. De même on remarque que le creux est plus aplatit, ce qui montre l'apport de la non linéarité de la houle.

\section{Calcul de l'évolution du tourbillon:}

On s'intéresse dans cette partie au calcul de la trajectoire d'une concentration tourbillonnaire dans la houle. Le domaine d'étude est illustré sur la figure (6).

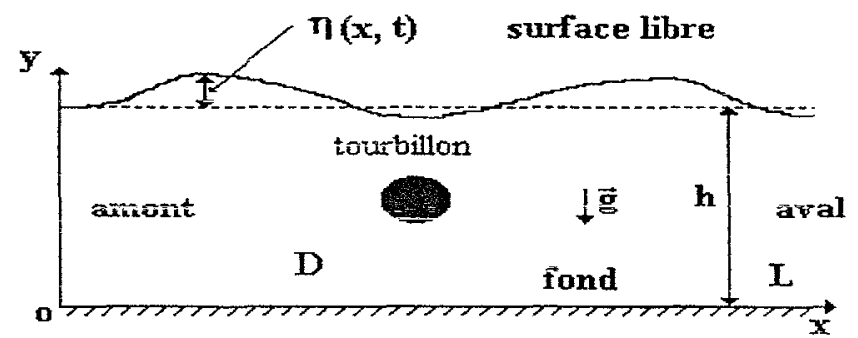

Figure (6) Schéma d'étude de l'évolution du tourbillouin

Le mouvement du tourbilion est régi par les équations de Navier Stokes écrites en terme de tourbillon $\mathrm{W}$ :

$$
\frac{\partial \vec{w}}{\partial t}+(\vec{U} \cdot \nabla) \vec{w}=v \Delta \vec{w}
$$

L'application de cette méthode à notre problème consiste à discrétiser le tourbillon concentré en un certain nômbre de particules quí seront suivies dans leur mouvement. Un opérateur de splitting permet de séparer l'équation du bilan du tourbillon en une équation représentant la diffusion (7) et une autre représentant la convection (8), soit respectivement:

$$
\frac{\partial \vec{w}}{\partial t}=v \Delta \vec{w} \quad \text { (7) } \quad \frac{d \vec{w}}{d t}=0 \text { et } \frac{d \vec{X}}{d t}=\vec{U}
$$

L'utilisation de l'intégrale de Biot Savart permet, en considérant les interactions mutuelles entre les particules, de déterminer leur vitesse, soit:

$$
\vec{U}(X)=\frac{1}{2 \pi} \iint_{R^{2}} \frac{\vec{w} \wedge \vec{X}}{\|\vec{X}\|^{2}} d \sigma
$$

Un schéma d'intégration en temps permet de résoudre l'équation (8) et de déterminer les positions des particules tourbillonnaires. L'intensité tourbillonnaire de chaque particule est calculée en considérant l'équation (7) de ce système. La méthode utilisée pour la résolution est celle de $S$. Huberson et J.P. Choquin [1] où la solution exacte de l'équation de la chaleur est utilisée pour la résolution avec en plus une technique permettant de tenir compte du tourbillon diffusé hors de chaque particule tourbillonnaire. Physiquement, cela revient à ne pas considérer les particules comme des particules ponctuelles, mais avec un certain étendu. Ceci permet d'une part de corriger la diffusion et d'autre part d'éviter les problèmes d'indétermination de la vitesse calculée par Biot Savart, car lorsque deux 
particules sont très proches, la vitesse devient infinie, ce qui est impossible physiquement.

Dans l'algorithme du couplage entre la houle et le tourbillon, on utilise une décomposition pour accéder à la vitesse globale de chaque particule. La première partie de cette solution contient l'effet du tourbillon et la seconde l'effet de la houle. La vitesse de la houle est déduite du potentiel $\varphi$ grâce à l'équation:

$$
\vec{U}_{\mathrm{h}}=\vec{\nabla} \varphi
$$

Ainsi on a la vitesse globale sous la forme:

$$
\vec{U}(X)=\frac{1}{2 \pi} \iint_{R^{2}} \frac{\vec{w} \wedge \vec{x}}{\|\vec{X}\|^{2}} \mathrm{~d} \sigma+\vec{U}_{h}
$$

\subsection{Validation du code de calcul de l'évolution du tourbillon:}

Pour valider cette démarche on a utilisé pour le calcul de la houle une solution analytique donnée par Stokes à l'ordre deux. L'objet est de mettre en oeuvre l'application de la méthode particulaire pour décrire l'évolution et la trajectoire d'une concentration tourbillonnaire dans la houle. Les paramètres de cette évolution sont: la viscosité $v$, la circulation du tourbillon $\Gamma$. On a choisi dans un premier temps le cas d'une houle de Stokes linéaire. On présente sur les figures 7 et 8 les résultats concernants la trajectoire de la particule représentant le centre du tourbillon concentré, dans le cas d'une circulation faible et une circulation importante.

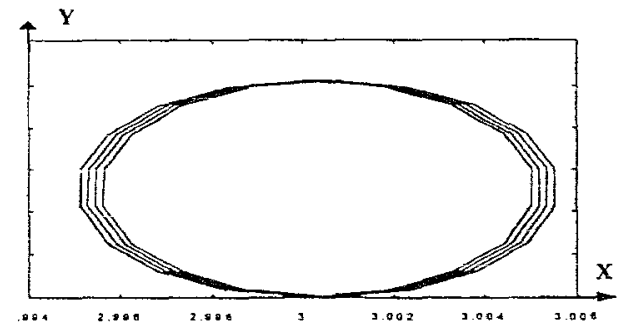

Figure7: $\Gamma=0.0001$

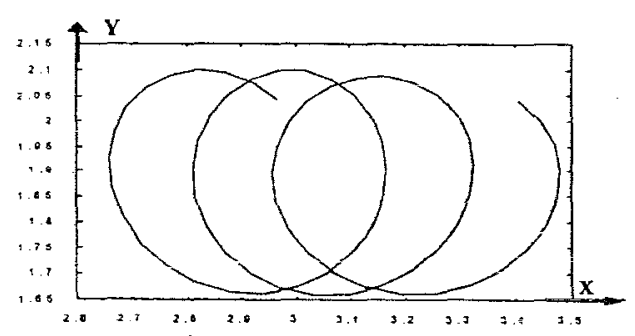

Figure8: $\Gamma=-1.5$

les coordonnées $X$ et $Y$ représentent respectivement l'abscisse et l'ordonnée du tourbilion. On constate la régularité spatiale de l'évolution du tourbillon. En effet lorsque le tourbillon reste faible, la trajectoire est fermée, et si on augmente la circulation le tourbillon est convecté. L'effet de la non linéarité de la houle apparait lorsqu'on change de cambrure (figure 9: zone intermédiaire et 10: zone peu profonde).
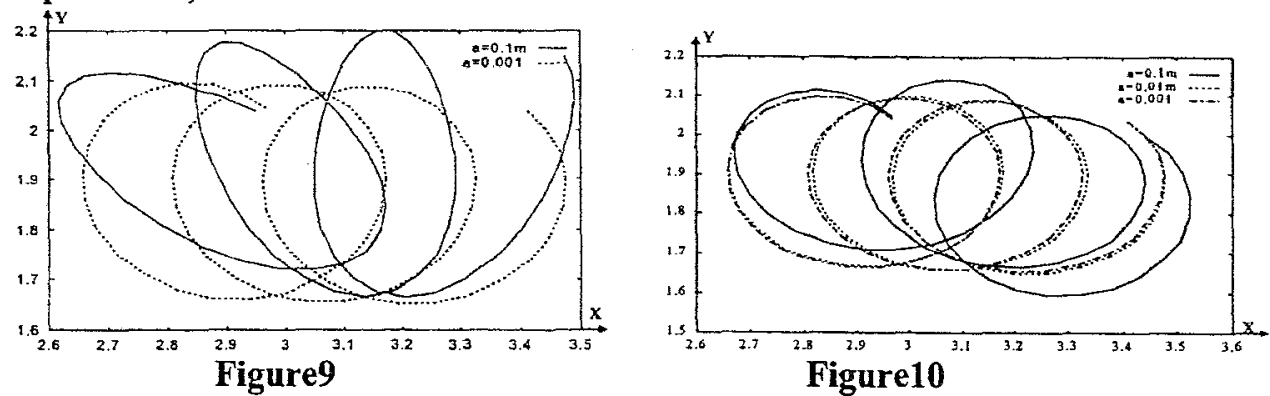

Figure10 
Les effets de la non linéarité se manifestent donc par une perte de symétrie sur la trajectoire du tourbillon et l'interaction avec le fond devient plus importante en particulier pour des amplitudes assez grandes.

\section{Le modèle houle-tourbillon: solution par Couplage numérique:}

Dans le cas où la houle est calculée numériquement, le problème se complique. En effet le champs de houle est obtenue dans un domaine discrétisé : (les valeurs du champs des vitesses dû à la houle sont calculés sur les noeuds du maillage) et le champs tourbillonnaire est calculé d'une manière Lagrangienne. Pour faire le couplage il faut d'abord localiser les particules dans le domaine discrétisé (sur le maillage), ensuite il faut faire la projection du champs de houle sur les particules tourbillonnaires. L'algorithme qu'on a utilisé est le suivant:

1) Initialisation (Position des particules, champs de houle). 2) Calcul du champs de houle (voir section 1). 3) Projection du champs particulaires sur le maillage. 4) Calcul du champs de vitesse induit par la houle sur la position des particules. 5) Calcul de la trajectoire et l'évolution du tourbillon (voir section 2). 6) Test sur le temps physique.

Cet algorithme a été mis en pratique, il nous a permis de retrouver les résultats illustrés sur la figure 7 et la figure 9 dans le cas où le tourbillon est placé initialement assez loin du fond et de la surface libre.

\section{4- Conclusion:}

Il nous reste à valider l'algorithme du couplage numérique houletourbillon dans des configurations complexes, en particulier lorsque le tourbillon se trouve proche de la surface libre ou du fond variable. D'autres problèmes seront abordés qui concernent la génération du tourbillon, le suivie dans le cas où il sort du domaine d'étude et le couplage dans le cas d'un fluide réel.

\section{Références:}

[1] S. Huberson - J. P. Choquin "Particles simulations of viscous flow" Computers and Fluids $N^{\circ} 2$, pp. $397-410 ., 1988$

[2] G. Mangoub, «Étude numérique de l'interaction tourbillon paroi par une méthode particules maillage», Thèse de Doctorat, Université Paris VI, Décembre 1992.

[3] S. Mordane "Calcul du problème de la houle non linéaire et instationnaire par une méthode asymptotique- numérique " Thèse $3^{\text {eme }}$ cycle, Université Hassan II Fac Sc Ben M'Sik Casablanca, 1995.

[4] J. Douglas, J. Gunn:"A General Formulation of Alternating Direction Methods" Numèrische mathèmatik 6, 428-453, 1964.

[5] Wachpress E.L: "Iterative solution of elliptic systems". Prentice Hall 1966.

[6]Orlansky, 1976:"A simple boundary condition for unbonded hyperbolic flows" J.C.P, 21,pp.251-269. 